\title{
Foreword
}

\author{
Anna Maria Bigatti • Francisco Botana • \\ Thierry Dana-Picard · Felipe Gago • \\ Ilias Kotsireas • Manuel Ladra • Wei Li
}

Published online: 13 November 2019

(C) Springer Nature Switzerland AG 2019

The Applications of Computer Algebra (ACA) conference series ${ }^{1}$ is devoted to promoting all kinds of computer algebra applications, and encouraging the interaction of developers of computer algebra systems and packages with researchers and users (including scientists, engineers, educators, and mathematicians). Topics include, but are not limited to, computer algebra in the sciences, engineering, communication, medicine, pure and applied mathematics, education and computer science.

The twenty-fourth conference in the series, ACA 2018, ${ }^{2}$ was held at University of Santiago de Compostela in Spain, June 18th-22nd, 2018. Manuel Ladra served as General Chair of ACA 2018, while Francisco Botana served as Program Chair.

The conference featured 4 invited speakers:

${ }_{1}^{1} \mathrm{http}: / /$ math.unm.edu/aca.html.

2 https://www.usc.es/regaca/aca2018.

A. M. Bigatti

DIMA, Università degli Studi di Genova, Genova, Italy

e-mail: bigatti@dima.unige.it

\section{F. Botana}

Department of Applied Mathematics I, University of Vigo, Pontevedra, Spain

e-mail: fbotana@uvigo.es

T. Dana-Picard

Jerusalem College of Technology, Jerusalem, Israel

e-mail: ndp@jct.ac.il

F. Gago $(\varangle) \cdot$ M. Ladra

Department of Mathematics, University of Santiago de Compostela, Santiago de Compostela, Spain

e-mail: felipe.gago@usc.es

M. Ladra

e-mail: manuel.ladra@usc.es

I. Kotsireas

Wilfrid Laurier University, Waterloo, Canada

e-mail: ikotsire@wlu.ca

W. $\mathrm{Li}$

KLMM, Academy of Mathematics and Systems Science, Chinese Academy of Sciences, Beijing, China e-mail: liwei@mmrc.iss.ac.cn 
- James H. Davenport (University of Bath, United Kingdom), who spoke on “Applications of Computer Algebra to Verification and Satisfiability Checking".

- Vijay Ganesh (University of Waterloo, Canada), who spoke on "SAT Solvers and Computer Algebra Systems: A Powerful Combination for Mathematics".

- Laureano Gonzlez-Vega (University of Cantabria, Spain), who spoke on "Dealing with real algebraic curves and surfaces for discovery: from experiments to theory and applications".

- Dingkang Wang (Chinese Academy of Sciences, People's Republic of China), who spoke on "Automatic Geometric Theorem Proving and Discovering using (Comprehensive) Groebner Bases".

The conference was structured as a series of special sessions:

- General Session

- Computer Algebra Modeling in Science and Engineering

- Computer Algebra in Education

- Applied and Computational Algebraic Topology

- Computer Algebra for Dynamical Systems and Celestial Mechanics

- Computational Differential and Difference Algebra

- Algebraic and Algorithmic Aspects of Differential and Integral Operators

- Dynamic Geometry and Mathematics Education

- Computer Algebra in Coding Theory and Cryptography

- Parametric Polynomial Systems

- Algorithms for Zero-Dimensional Ideals

- Numerical Differential and Polynomial Algebra

The conference book of abstracts was published by the Servizo de Publicacións e Intercambio Científico ${ }^{3}$ of the University of Santiago de Compostela.

This special issue of Mathematics in Computer Science (MCS) contains 27 formally refereed articles accepted from 38 submissions of full papers presented (or related to presentations) at ACA 2018.

We wish to express our sincere thanks to the anonymous referees who provided constructive and detailed referee reports that helped the authors of accepted papers to improve them substantially. Without their hard work it would not have been possible to produce the current MCS special issue.

During the last part of the editing proccess our thoughts were, and still are, with our friend Francisco Botana and with his family. We wish him the very best during his recovery and that this time help him to recuperate and re-energize.

Publisher's Note Springer Nature remains neutral with regard to jurisdictional claims in published maps and institutional affiliations.

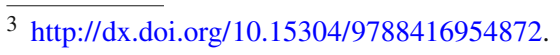

